\title{
A Multi-Scale Geometric Flow for Segmenting Vasculature in MRI
}

\author{
Maxime Descoteaux ${ }^{1}$, Louis Collins $^{2}$, and Kaleem Siddiqi ${ }^{1}$ \\ McGill University \\ Montréal, QC, Canada \\ ${ }^{1}$ School of Computer Science \& Centre For Intelligent Machines \\ \{mdesco, siddiqi\}@cim.mcgill.ca \\ ${ }^{2}$ McConnell Brain Imaging Centre, Montréal Neurological institute \\ \{louis\}@bic.mni.mcgill.ca
}

\begin{abstract}
Often in neurosurgical planning a dual echo acquisition is performed that yields proton density (PD) and T2-weighted images to evaluate edema near a tumour or lesion. The development of vessel segmentation algorithms for PD images is of general interest since this type of acquisition is widespread and is entirely noninvasive. Whereas vessels are signaled by black blood contrast in such images, extracting them is a challenge because other anatomical structures also yield similar contrasts at their boundaries. In this paper we present a novel multi-scale geometric flow for segmenting vasculature from PD images which can also be applied to the easier cases of computed tomography (CT) angiography data or Gadolinium enhanced MRI. The key idea is to first apply Frangi's vesselness measure [4] to find putative centerlines of tubular structures along with their estimated radii. This multi-scale measure is then distributed to create a vector field which is orthogonal to vessel boundaries so that the flux maximizing flow algorithm of [17] can be applied to recover them. We validate the approach qualitatively with PD, angiography and Gadolinium enhanced MRI volumes.
\end{abstract}

\section{Introduction}

A three-dimensional (3D) representation of vasculature can be extremely important in image-guided neurosurgery, pre-surgical planning and clinical analysis. It is unfortunately often the case that in order to obtain such representations from an MRI volume an expert has to interact with it manually slice by slice while colouring regions of interest and connecting them using image processing operations. This process is extremely laborious, is prone to human error and makes large scale clinical studies of vasculature infeasible. In computer vision there has been a significant amount of work towards automating the extraction of vessels or vessel centerlines. Whereas an exhaustive review of this literature is beyond the scope of this article, typical examples include: 1) active contours or surfaces for angiography data [13], 2) multi-scale methods for model-based segmentation of tubular structures [9], 3) statistical methods which use mixture models [18], and 4) methods based on intensity ridge detection and traversal [8, 3]. It should be pointed out that most of these methods have been demonstrated for 2D projection angiography, 3D CT angiography or Gadolinium enhanced MRI, and these modalities can 


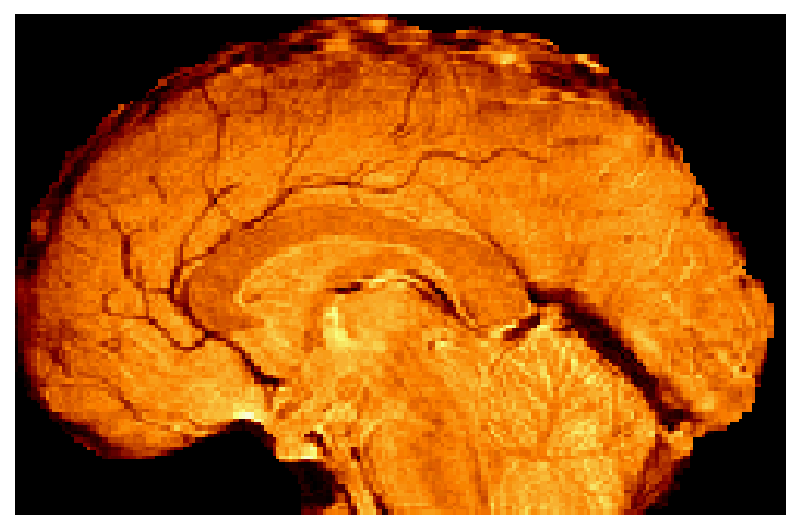

Fig. 1. A sagittal view of a proton density (PD) weighted MRI volume acquired at the Montreal Neurological Institute. The spaghetti-like structures correspond to vasculature.

require the injection of contrast agents. To our knowledge no method currently exists for the automatic extraction of vessel boundaries in standard MRI volumes such as the PD image shown in Figure 1. Here it is clear that a signal decrease is present in the vascular regions (the spaghetti-like structures), but the contrast between blood vessel and surrounding tissue is not as great when compared to the angiographic sequences. Hence, the problem of recovering vessels from image intensity contrast alone on PD-weighted images is a challenge and requires shape information to constrain the segmentation. If successful, such a procedure could result in a vascular model that could be used in surgical planning while eliminating the need for an additional scan thus saving time during image acquisition and easing the burden on the patient.

In this paper we introduce a novel algorithm for vessel segmentation which is designed for the PD images, but can be applied as well to angiographic data or Gadolinium enhanced MRI volumes. The algorithm is motivated in part by the approach in [15] where Frangi's vesselness measure [4] is thresholded to find centerlines. Tubular fits to vessel boundaries are then obtained using a form of connected component analysis and a generalized cylinder model. This latter step typically yields results that are disconnected. In our approach the vesselness measure is extended to yield a vector field which is locally normal to putative vessel boundaries. This in turn allows the flux maximizing geometric flow of [17] to be applied, which has a formal motivation, is topologically adaptive due to its implementation using level set methods, and finally is computationally efficient. We illustrate the power of this geometric-flow based framework with segmentation results on PD data, and also illustrate its applicability to Gadolinium enhanced MRI volumes and angiography data.

The paper is outlined as follows. In Section 2 we review relevant background material on geometric flows for vessel segmentation and on the use of the Hessian matrix to model tubular structures. We then develop our multi-scale geometric flow by incorporating Frangi's vesselness measure [4] in the flux maximizing flow algorithm of [17] in 
Section 3. We present several reconstruction results to validate the algorithm in Section 4 and conclude by discussing ongoing work in Section 5 .

\section{Background}

\subsection{Geometric flows}

In the context of geometric flows for segmenting vasculature, there are two recent approaches which are relevant to the development here. First, Lorigo et al. propose a regularization of a geometric flow in 3D using the curvature of a 3D curve [12]. This approach is grounded in the recent level set theory developed for mean curvature flows in arbitrary co-dimension [2]. It yields a flow which is designed to recover vessel boundaries signaled by the gradient in angiography data, while under the influence of a smoothing term driven by the mean curvature of an implied centerline. Second, Vasilevskiy and Siddiqi derive the gradient flow which evolves a curve (2D) or a surface (3D) so as to increase the inward flux of a fixed (static) vector field through its boundary as fast as possible [17]. With $S$ an evolving surface and $\overrightarrow{\mathcal{V}}$ the vector field, this flow is given by

$$
\mathcal{S}_{t}=\operatorname{div}(\overrightarrow{\mathcal{V}}) \overrightarrow{\mathcal{N}}
$$

where $\overrightarrow{\mathcal{N}}$ is the unit inward normal to each point on $S$. This flow evolves a surface to a configuration where its normals are aligned with the vector field. In the context of segmenting vasculature in angiographic images, $\overrightarrow{\mathcal{V}}$ can be selected to be the gradient of the intensity image which is expected to be orthogonal to vessel boundaries.

A limitation of both the above approaches is that they are designed specifically for angiographic data and hence require restrictive assumptions to hold:

1. Both methods are initialized essentially by thresholding such data, and thus would fail when vessel boundaries cannot be identified from contrast alone.

2. Neither approach has an explicit term to model tubular structures. Rather, each flow relies on the assumption that the gradient of the intensity image yields a quantity that is significant only at vessel boundaries.

3. Neither of these methods takes into account explicitly the multi-scale nature of vessels boundaries as they appear in all modalities.

In this paper we overcome several of these limitations by incorporating a measure of "vesselness" based on the Hessian matrix.

\subsection{Modeling vasculature using the Hessian}

Several multi-scale approaches to modeling tubular structures in intensity images have been based on properties of the Eigen values of the Hessian matrix $\mathbf{H}[11,16,4]$. These methods exploit the fact that at locations centered within tubular structures the smallest Eigen value of $\mathbf{H}$ is close to zero (reflecting the low curvature along the direction of the vessel) and the two other Eigen values are high and are close to being equal, reflecting the fact that the cross-section of the vessel is approximately circular. The corresponding 
Eigen vectors span the vessel direction and the cross-sectional plane. The Eigen value analysis can be pushed further to differentiate tube-like, blob-like, sheet-like, and noiselike structures from one another as summarized in Table 1.

\begin{tabular}{l|l|l} 
Eigen value conditions & local structure & examples \\
\hline$\lambda_{1} \approx 0, \lambda_{2} \approx \lambda_{3}>>0$ & tube-like & vessel, bronchus \\
$\lambda_{1} \approx \lambda_{2} \approx 0, \lambda_{3}>>0$ & sheet-like & cortex, skin \\
$\lambda_{1} \approx \lambda_{2} \approx \lambda_{3}>>0$ & blob-like & nodule \\
$\lambda_{1} \approx \lambda_{2} \approx \lambda_{3} \approx 0$ & noise-like & noise
\end{tabular}

Table 1. A classification of local structures based on the Eigen values of the Hessian matrix. Here, we assume that $\left|\lambda_{1}\right| \leq\left|\lambda_{2}\right| \leq\left|\lambda_{3}\right|$. The sign of the highest Eigen values generally indicate whether the local structure is dark on a bright background or bright on a dark background. A positive sign corresponds to a dark structure on a bright background which is the case for PD weighted MRI volumes.

We choose to focus here on Frangi's vesselness measure [4] because it incorporates information from all three Eigen values. Three quantities are defined to differentiate blood vessels from other structures:

$$
R_{B}=\frac{\left|\lambda_{1}\right|}{\sqrt{\left|\lambda_{2} \lambda_{3}\right|}}, \quad R_{A}=\frac{\left|\lambda_{2}\right|}{\left|\lambda_{3}\right|}, \quad S=\sqrt{\lambda_{1}^{2}+\lambda_{2}^{2}+\lambda_{3}^{2}}
$$

From Table 1, it can be seen that $R_{B}$ distinguishes blob-like structures from other patterns. The $R_{A}$ ratio differentiates sheet-like from tube-like structures. Finally, $S$, the Frobenius norm, is used to ensure that random noise effects are suppressed from the response. For a particular scale $\sigma$ the intensity image is first convolved by a Gaussian at that scale, $G(\sigma)$ following which the vesselness measure is defined by ${ }^{1}$.

$$
V(\sigma)=\left\{\begin{array}{ll}
0 & \text { if } \lambda_{2}<0 \text { or } \lambda_{3}<0 \\
\left(1-\exp \left(-\frac{R_{A}^{2}}{2 \alpha^{2}}\right)\right) \exp \left(-\frac{R_{B}^{2}}{2 \beta^{2}}\right)\left(1-\exp \left(-\frac{S^{2}}{2 c^{2}}\right)\right)
\end{array}\right\}
$$

This measure is designed to be maximum along the centerlines of tubular structures and close to zero outside vessel-like regions. In our implementation we set the parameters $\alpha, \beta$ and $c$ to $0.5,0.5$ and half the maximum Frobenuis norm, respectively, as suggested in $[4,15]$. At each voxel we compute vesselness responses using ten log scale increments between $\sigma=0.2$ and $\sigma=2.0$ (in our data the maximum radius of a vessel is 2 voxels) and select the maximum vesselness response along with its scale. The chosen scale gives the estimated radius of the vessel and the Eigen vector associated with the smallest Eigen value its local orientation.

\footnotetext{
${ }^{1}$ In practice we directly compute the entries which comprise the Hessian matrix by using derivatives of Lindeberg's $\gamma$-parametrized normalized Gaussian kernels [10], which allows us to compare responses at different scales.
} 

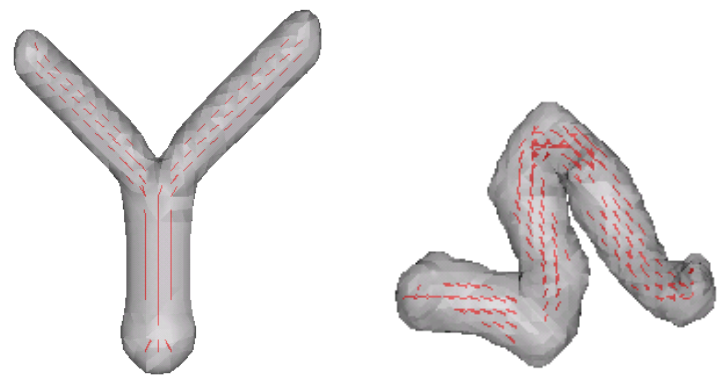

Fig. 2. A synthetic Y-shaped structure and a synthetic helix. For each structure the red vectors indicate the estimated vessel orientation at locations where the multi-scale vesselness measure (Eq. 2) is high.

This process is illustrated in Figure 2 for a synthetic Y-shaped structure and a synthetic helix. The grey surface coincides with a particular level set of the vesselness measure (which quickly drops to zero away from centerline locations). Within this surface locations of high vesselness are indicated by overlaying the Eigen vectors which correspond to the estimated vessel orientation. Observe that locations of high vesselness are close to vessel centerlines, and that the estimated vessel orientation at these locations is accurate. This information along with the estimated radius of associated vessels can be used to construct an appropriate vector field to drive the flux maximizing geometric flow, as we shall see in the following section.

\section{A Multi-Scale Geometric Flow For Segmenting Vasculature}

Our goal now is to extend the flux maximizing flow algorithm of [17] so that the vector field which drives it lies along the surface of putative vessel boundaries. This allows us to lift many of the restrictions on the flow pointed out in Section 2, because an explicit model of a tubular structure is now incorporated along with an appropriate notion of scale.

\subsection{Construction of the vector field}

The key idea is to distribute the vesselness measure, which is concentrated at centerlines, to the vessel boundaries which are implied. At each voxel $(x, y, z)$ where the vesselness measure is a local maximum in a $3 \times 3 \times 3$ neighborhood we consider an ellipsoid with its major axis aligned with the estimated orientation and its two semi-minor axes equal to the estimated radius. In our implementation the semi-major axis length is chosen to be twice that of the semi-minor axes. The vesselness measure is then distributed over every voxel $\left(x_{e}, y_{e}, z_{e}\right)$ on the boundary of the ellipsoid by scaling it by the projection of the vector from $(x, y, z)$ to $\left(x_{e}, y_{e}, z_{e}\right)$ onto the cross-sectional plane, as illustrated in Figure 3. If $(x, y, z)$ is taken to be the origin $(0,0,0)$ and the $x y$ 


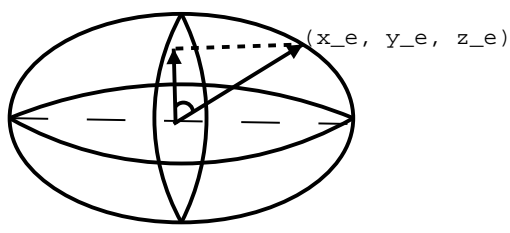

(1)

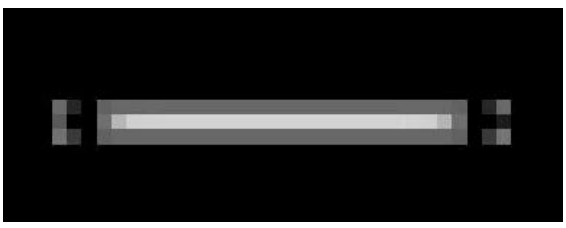

(3)

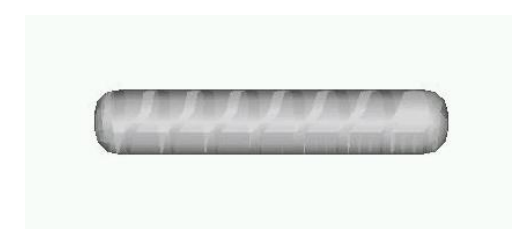

(2)

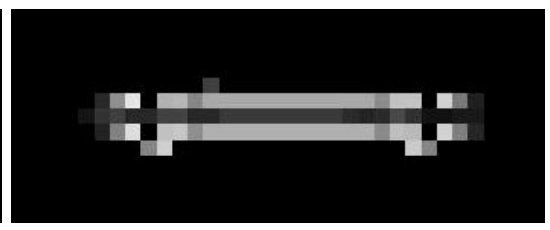

(4)

Fig. 3. Distributing the vesselness measure to the implied boundaries. (1) The vector from the center of the ellipsoid to the surface voxel $\left(x_{e}, y_{e}, z_{e}\right)$, as well as its projection onto the crosssectional plane, taken to be the $x y$ plane. We distribute the vesselness measure to all $\left(x_{e}, y_{e}, z_{e}\right)$ on the ellipsoid by scaling it by the magnitude of this projection. (2) A synthetic tube of radius 2. (3) A view of the vesselness measure in a slice. Brighter regions indicate stronger vesselness. (4) A view of the $\phi$ distribution in the same slice. As expected, we have local maxima of the vesselness measure on the centerline in (3) and local maxima at the boundaries of the tube on the $\phi$ distribution in (4).

plane is taken to coincide with the cross-sectional plane this scale factor works out to be $\left\langle\left(x_{e}, y_{e}, z_{e}\right), \frac{\left(x_{e}, y_{e}, 0\right)}{\sqrt{x_{e}^{2}+y_{e}^{2}}}\right\rangle=\sqrt{x_{e}^{2}+y_{e}^{2}}$. This process of distributing the vesselness measure to the implied boundaries clearly favours voxels in the cross-sectional plane. We define the addition of the extensions carried out independently at all voxels to be the $\phi$ distribution. The extended vector field is now defined as the product of the normalized gradient of the original image with the above $\phi$ distribution

$$
\overrightarrow{\mathcal{V}}=\phi \frac{\nabla \mathcal{I}}{|\nabla \mathcal{I}|}
$$

This vector field embodies two important constraints. First, the magnitude of $\phi$ is maximum on vessel boundaries and the ellipsoidal extension performs a type of local integration ${ }^{2}$. Second, $\frac{\nabla \mathcal{I}}{|\nabla \mathcal{I}|}$ captures the direction information of the gradient, which is expected to be high at boundaries of vessels as well as orthogonal to them, which is the basic motivation for the flux maximizing geometric flow. It is important to normalize the gradient of the image so that its magnitude does not dominate the measure in regions of very low vesselness. For example, structures such as white and gray matter boundaries could then get significant unwanted contributions.

\footnotetext{
${ }^{2}$ This follows because the local maximum vesselness criterion enforces the condition that the extension is carried out only from locations as close as possible to vessel centerlines.
} 


\subsection{The multi-scale geometric flow}

The extended vector field explicitly models the scale at which vessel boundaries occur, due to the multi-scale nature of the vesselness measure $V(\sigma)$ (Eq. 2) as well as the expected gradient in the direction normal to vessel boundaries. Thus, it is an ideal candidate for the static vector field in the flux maximizing geometric flow (Eq. 1). The surface evolution equation then works out to be

$$
\begin{aligned}
S_{t} & =\operatorname{div}(\overrightarrow{\mathcal{V}}) \overrightarrow{\mathcal{N}} \\
& =\left[\left\langle\nabla \phi, \frac{\nabla \mathcal{I}}{|\nabla \mathcal{I}|}\right\rangle+\phi \operatorname{div}\left(\frac{\nabla \mathcal{I}}{|\nabla \mathcal{I}|}\right)\right] \overrightarrow{\mathcal{N}} \\
& =\left[\left\langle\nabla \phi, \frac{\nabla \mathcal{I}}{|\nabla \mathcal{I}|}\right\rangle+\phi \kappa_{\mathcal{I}}\right] \overrightarrow{\mathcal{N}}
\end{aligned}
$$

where $\kappa_{\mathcal{I}}$ is the Euclidean mean curvature of the iso-intensity level set of the image. Note that this is a hyperbolic partial differential equation since all terms depend solely on the vector field and not on the evolving surface. We now enumerate several properties of this geometric flow.

1. The first term $\left\langle\nabla \phi, \frac{\nabla \mathcal{I}}{|\nabla \mathcal{I}|}\right\rangle$ acts like a doublet. $\nabla \phi$ has a zero-crossing at vessel boundaries and $\nabla \mathcal{I}$ does not change sign. Hence, when the evolving surface overshoots the boundary slightly, this term acts to push it back towards the boundary.

2. The second term behaves like a geometric heat equation since $\kappa_{\mathcal{I}}$ is the mean curvature of the iso-intensity level set of the original intensity image. This equation has been extensively studied in the mathematics literature and has been shown to have remarkable smoothing properties $[5,6]$. It is also the basis for several nonlinear geometric scale-spaces such as those studied in $[1,7]$.

3. Combining both terms, it is clear that the flow cannot leak in regions outside vessels since both $\phi$ and $\nabla \phi$ are zero there. Hence, when seeds are placed at locations where the vesselness measure $V(\sigma)$ is high the flow given by Eq. 3 will smoothly evolve toward zero level set of the divergence of the vector field $\vec{V}$.

\subsection{Implementation Details}

Below we review some of the details of the implementation of our multi-scale geometric flow (Eq. 3), which is based on level set methods [14].

1. We run five iterations of mean curvature type smoothing on the original image, which is a standard method to remove artifacts such as speckle noise since it smooths along iso-intensity level sets but not across them.

2. We compute the Hessian operator over $10 \log$ scales and select the maximum vesselness response as described in Section 2. We use Jacobi's method for symmetric matrices to find Eigen values of the Hessian.

3. The $\phi$ distribution in Section 3.1 is carried out from voxels at vessel centerlines since at such locations one has strong confidence in the scale and orientation estimate from Frangi's vesselness measure [4]. This is done using the following procedure

$$
\begin{aligned}
& \text { if }\left(V(\sigma)>\text { threshold \&\& } \frac{V(\sigma)}{\text { local_max }}>\text { percentile }\right) \\
& \text { Distribute vesselness over ellipsoid }
\end{aligned}
$$


For all examples we use a vesselness threshold of 0.01 and a percentile of 0.75 and local_max is the maximum vesselness response in a $3 \times 3 \times 3$ neighborhood of the voxel.

4. The doublet term $\left\langle\nabla \phi, \frac{\nabla \mathcal{I}}{|\nabla \mathcal{I}|}\right\rangle$ is computed using central differences for the first term and a second-order essentially non-oscillatory (ENO) scheme for the second.

5. $\kappa_{\mathcal{I}}$, the mean curvature of each intensity iso-surface is computed using a 3-neighbor central difference scheme for all derivatives:

$$
\kappa_{\mathcal{I}}=\frac{\left(\mathcal{I}_{y y}+\mathcal{I}_{z z}\right) \mathcal{I}_{x}^{2}+\left(\mathcal{I}_{x x}+\mathcal{I}_{z z}\right) \mathcal{I}_{y}^{2}+\left(\mathcal{I}_{x x}+\mathcal{I}_{y y}\right) \mathcal{I}_{z}^{2}-2\left(\mathcal{I}_{x} \mathcal{I}_{y} \mathcal{I}_{x y}+\mathcal{I}_{x} \mathcal{I}_{z} \mathcal{I}_{x z}+\mathcal{I}_{y} \mathcal{I}_{z} \mathcal{I}_{y z}\right)}{\left(\mathcal{I}_{x}^{2}+\mathcal{I}_{y}^{2}+\mathcal{I}_{z}^{2}\right)^{\frac{3}{2}}}
$$

6. A first-order in time discretized form of the level-set version of the evolution equation is given by

$$
\Psi_{n}=\Psi_{n-1}+\Delta t * \mathcal{F} *\left\|\nabla \Psi_{n-1}\right\|
$$

where $\mathcal{F}=\left\langle\nabla \phi, \frac{\nabla \mathcal{I}}{|\nabla \mathcal{I}|}\right\rangle+\phi \operatorname{div}\left(\frac{\nabla \mathcal{I}}{|\nabla \mathcal{I}|}\right), \Psi$ is the embedding hypersurface and $\Delta t$ is the step size. This is now a standard numerical approach for solving partial differential equations of this type since it allows topological changes to occur without any additional computational complexity and can be made efficient using a narrow band implementation. The evolving surface $S$ is obtained as the zero level set of the $\Psi$ function. The numerical derivatives used to estimate $\|\nabla \Psi\|$ must be computed with up-winding in the proper direction as described in [14].

\section{Examples}

We illustrate our multi-scale geometric flow for segmenting vasculature on three different modalities: MRA, Gadolinium enhanced MRI and PD. The same parameters were used throughout as described in Section 3.3 with the exception that for the PD data the vesselness threshold was lowered to 0.005 and the numerical time step was lowered to 0.5 in order to capture smaller structures. We should point out that whereas prior geometric flow based methods $[12,17]$ could be applied to the angiography data set, they would fail entirely on the latter two modalities where high contrast regions are not limited to vessel boundaries.

Figure 4 shows iterations of the flow using three single voxel seeds on an MRA data set obtained from the Montreal Neurological Institute (MNI). The flow is able to pick up the main vessels automatically. Several of the finer vessels are less than one voxel wide and hence a super-sampling strategy would have to be applied in a preprocessing step to the data in order to recover them.

Figure 5 depicts a $40 \mathrm{~mm} \times 53 \mathrm{~mm} \times 91 \mathrm{~mm}$ region centered on the corpus callosum from a Gadolinium enhanced MRI volume obtained at the MNI. The $1 \mathrm{~mm}$ isotropic data was super-sampled to $0.33 \mathrm{~mm}$ using a tricubic interpolation kernel, because several vessels in the original data set were less than one voxel wide. In the image one can see the callosal and supra-callosal arteries (the long arching vessels running from left to right). We show an MIP of a sagittal and a transverse view in the top row. A segmentation obtained by thresholding the vesselness map, as carried out in [15], is shown in 


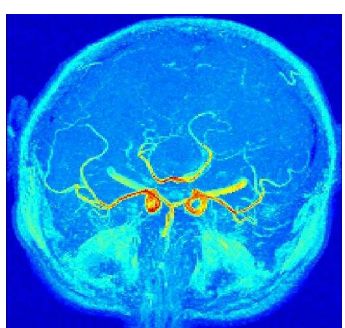

MIP

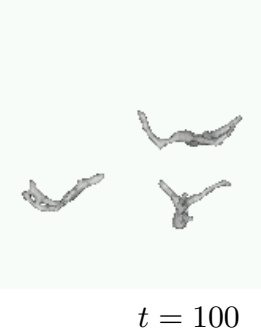

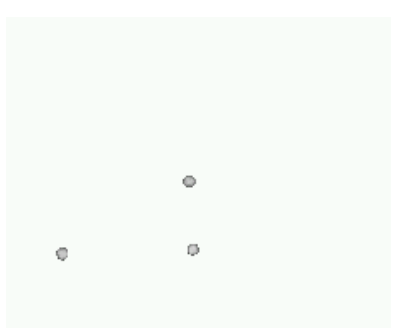

$t=0$

$t=55$

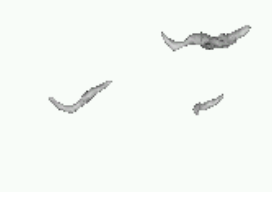

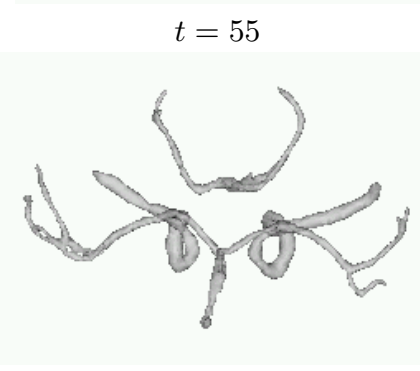

$t=2000$

Fig. 4. An illustration of the multi-scale geometric flow on a 68 x 256 x 256 MRA image. An MIP of the data is shown at the top left and the other images depict different stages of the evolution from three seeds.

the second row. This results in many disconnected vessels as well as artifacts. Our segmentation is shown in the third row and results in the reconstruction of well connected tubular structures.

Finally, Figure 6 depicts the segmentation of the full proton density MRI volume shown in Figure 1, which is clearly a challenge for most vessel segmentation algorithms. The PD data is acquired with $2 \mathrm{~mm}$ transverse slices with $1 \mathrm{~mm} \times 1 \mathrm{~mm}$ in-plane voxels. For this data set an MIP of the original data would not correspond to vasculature. Hence we choose to show MIPs of a sagittal and a transverse view of the vesselness measure in the top row. We then show the corresponding reconstructions obtained by our flow in the second row. A movie animating these segmentation results is available from the authors' research pages. To our knowledge, this is the first segmentation of a PD weighted MRI performed by a geometric flow in the literature. The reconstruction does not recover some of the finer vessels located near the surface of the brain, but these could be recovered using a finer placement of seeds along with an adaptive lowering of the vesselness threshold in those regions.

\section{Conclusions}

We have presented what to our knowledge is the first multi-scale geometric flow that can be applied for segmenting vasculature in PD weighted MRI volumes. The key idea is to incorporate a multi-scale vesselness measure in the construction of an appropriate vector field for a geometric flow. We have validated the flow qualitatively on several 


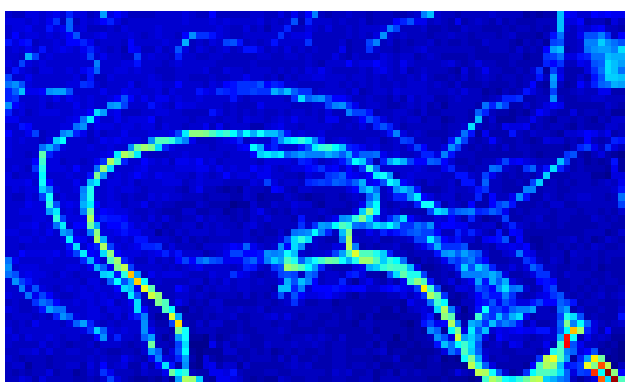

(1)

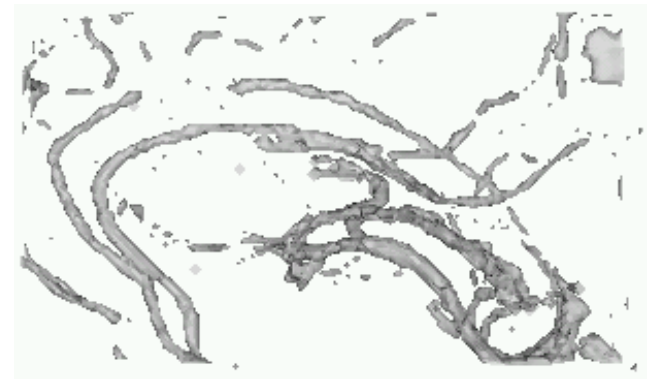

(3)

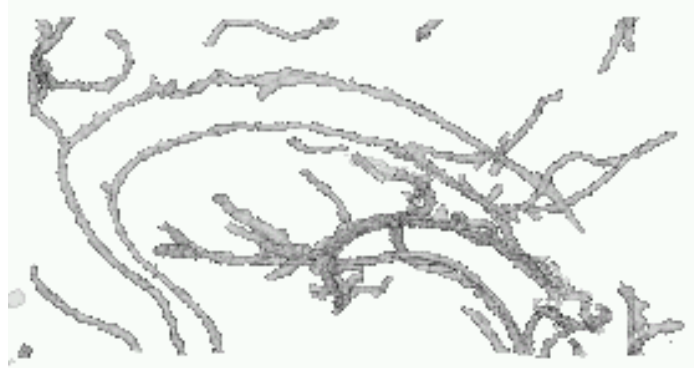

(5)

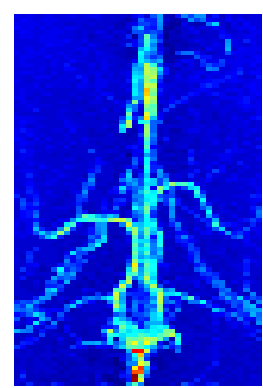

(2)

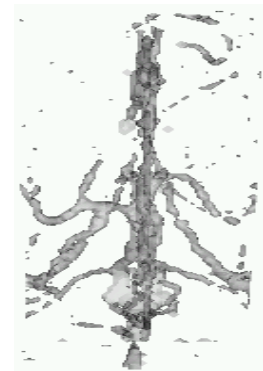

(4)

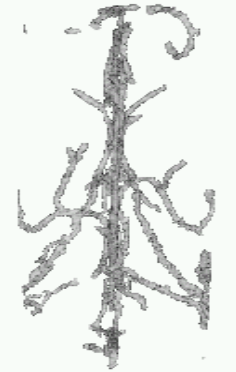

(6)

Fig. 5. An illustration of the flow on a $40 \mathrm{~mm}$ x $53 \mathrm{~mm}$ x $91 \mathrm{~mm}$ cropped region of a Gadolinium enhanced MRI. An MIP of the sagittal and transverse views of the data is shown in (1) and (2). Reconstructions obtained by simple thresholding for the same views are shown in (3) and (4). These are clearly sensitive to noise and result in disconnected or missing vessels. The results obtained by the multi-scale geometric flow are shown in (5) and (6). Observe that the flow has connected a section of the callosal arteries which is barely visible in the MIP (see (1),(3),(5)).

modalities. In particular, we have shown that a significant amount of vasculature can be recovered by initializing the flow using a few isolated seeds. In our experience we have also found that finer vessels can also be recovered by the manual placement of seeds by a user along with an adaptive lowering of the vesselness threshold used in the construction of the extended vector field $\overrightarrow{\mathcal{V}}$. 


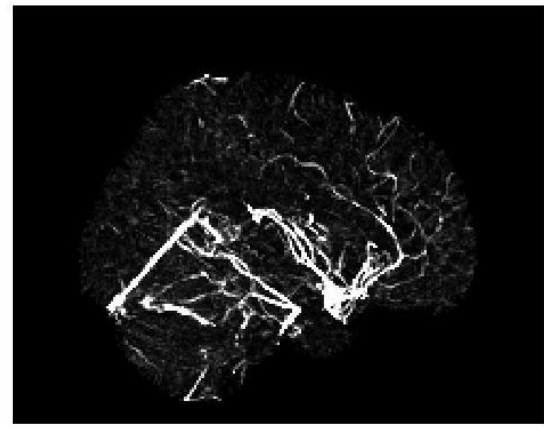

(1)

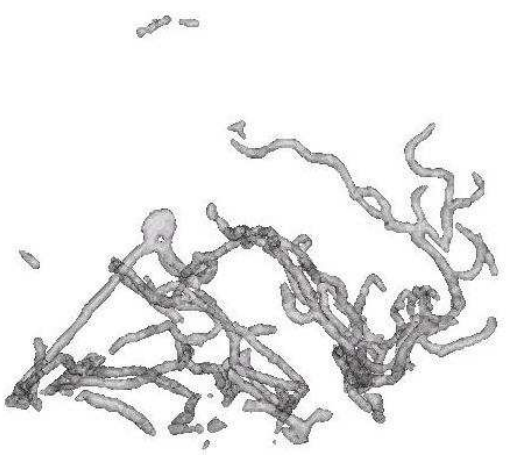

(3)

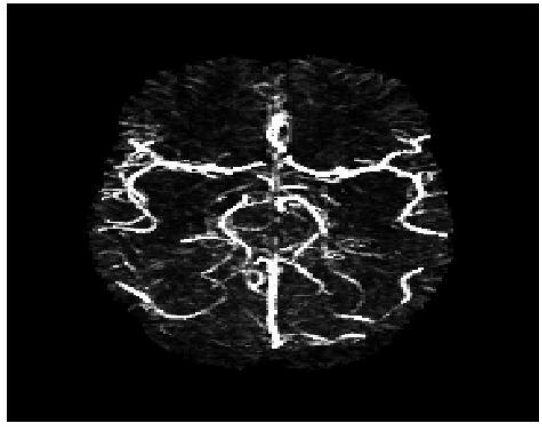

(2)

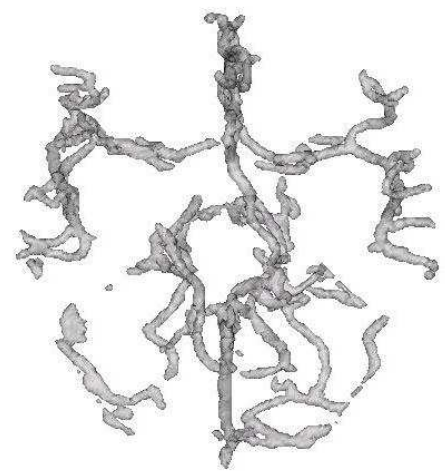

(4)

Fig. 6. An illustration of the flow on the full $181 \times 217 \times 181$ proton density weighted MRI volume of Figure 1. We show MIPs of the vesselness measure $V(\sigma)$ for sagittal and transverse views in (1) and (2), where we have masked the outside skull and the skin. The reconstructions obtained using our geometric flow with 10 manually placed seeds are shown in the bottom row. A movie illustrating this segmentation is included with this submission

In ongoing work we are carrying out a careful quantitative validation of the approach by acquiring data using different modalities on the same subject. This will allow us to evaluate the algorithm by using the results obtained on angiographic data as the ground truth. Once we have been able to fine tune its performance it is our hope that our implementation of this flow will become a basic image analysis tool for segmenting vasculature in clinical studies.

Acknowledgements This work was supported by grants from NSERC, FQRNT, CFI and CIHR. We are grateful to the reviewers for their helpful comments. 


\section{References}

1. L. Alvarez, F. Guichard, P. L. Lions, and J. M. Morel. Axiomes et équations fondamentales du traitement d'images. C. R. Acad. Sci. Paris, 315:135-138, 1992.

2. L. Ambrosio and H. M. Soner. Level set approach to mean curvature flow in arbitrary codimension. Journal of Differential Geometry, 43:693-737, 1996.

3. S. R. Aylward and E. Bullitt. Initialization, noise, singularities, and scale in height ridge traversal for tubular object centerline extraction. IEEE Transactions On Medical Imaging, 21(2):61-75, 2002.

4. A. Frangi, W. Niessen, K. L. Vincken, and M. A. Viergever. Multiscale vessel enhancement filtering. In MICCAI'98, pages 130-137, 1998.

5. M. Gage and R. Hamilton. The heat equation shrinking convex plane curves. Journal of Differential Geometry, 23:69-96, 1986.

6. M. Grayson. The heat equation shrinks embedded plane curves to round points. Journal of Differential Geometry, 26:285-314, 1987.

7. B. B. Kimia, A. Tannenbaum, and S. W. Zucker. Shape, shocks, and deformations I: The components of two-dimensional shape and the reaction-diffusion space. International Journal of Computer Vision, 15:189-224, 1995.

8. T. M. Koller, G. Gerig, G. Székely, and D. Dettwiler. Multiscale detection of curvilinear structures in 2-d and 3-d image data. In International Conference On Computer Vision, pages 864-869, 1995.

9. K. Krissian, G. Malandain, and N. Ayache. Model-based detection of tubular structures in 3d images. Computer Vision and Image Understanding, 80(2):130-171, November 2000.

10. T. Lindeberg. Edge detection and ridge detection with automatic scale selection. International Journal of Computer Vision, 30(2):77-116, 1998.

11. C. Lorenz, I. Carlsen, T. Buzug, C. Fassnacht, and J. Weese. Multi-scale line segmentation with automatic estimation of width, contrast and tangential direction in $2 \mathrm{~d}$ and $3 \mathrm{~d}$ medical images. In CVRMED-MRCAS'97, Lecture Notes in Computer Science, volume 1205, pages 233-242, 1997.

12. L. M. Lorigo, O. D. Faugeras, E. L. Grimson, R. Keriven, R. Kikinis, A. Nabavi, and C.-F. Westin. Curves: Curve evolution for vessel segmentation. Medical Image Analysis, 5:195206, 2001.

13. T. McInerney and D. Terzopoulos. T-snakes: Topology adaptive snakes. Medical Image Analysis, 4:73-91, 2000.

14. S. J. Osher and J. A. Sethian. Fronts propagating with curvature dependent speed: Algorithms based on hamilton-jacobi formulations. Journal of Computational Physics, 79:12-49, 1988.

15. L. Ostergaard, O. Larsen, G. Goualher, A. Evans, and D. Collins. Extraction of cerebral vasculature from mri. In 9th Danish Conference on Pattern Recognition and Image Analysis, August 2000.

16. Y. Sato, S. Nakajima, N. Shiraga, H. Atsumi, S. Yoshida, T. Koller, G. Gerig, and R. Kikinis. $3 \mathrm{~d}$ multi-scale line filter for segmentation and visualization of curvilinear structures in medical images. Medical Image Analysis, 2(2):143-168, 1998.

17. A. Vasilevskyi and K. Siddiqi. Flux maximizing geometric flows. IEEE Transactions on Pattern Analysis and Machine Intelligence, 24(12):1-14, 2002.

18. D. L. Wilson and A. Noble. Segmentation of cerebral vessels and aneurysms from $\mathrm{mr}$ aniography data. In Information Processing in Medical Imaging, pages 423-428, 1997. 